\title{
The debut of a rational treatment for an inherited neuropathy?
}

\author{
Steven S. Scherer
}

Department of Neurology, The Perelman School of Medicine at the University of Pennsylvania, Philadelphia, Pennsylvania, USA.

\begin{abstract}
Hereditary neuropathies are common neurological conditions characterized by progressive loss of motor and/or sensory function. There are no effective treatments. Among the many causes of hereditary neuropathies are dominant mutations in serine palmitoyltransferase, long chain base subunit 1 (SPTLC1), which cause hereditary sensory and autonomic neuropathy type 1 (HSAN1). By incorporating $\mathrm{L}$-alanine in place of $\mathrm{L}$-serine, the mutant HSAN1-associated serine palmitoyltransferase generates deoxysphingolipids, which are thought to be neurotoxic. In this issue of the JCI, Garofalo and colleagues report that oral L-serine reverses the accumulation of deoxysphingolipids in humans with HSAN1 and in a transgenic mouse model. As oral L-serine reduces the severity of neuropathy in the mouse model of HSAN1, these data suggest a rational candidate therapy for this devastating condition.
\end{abstract}

The peripheral nerves are the specialized conduits for the axons that connect the brain and spinal cord to the rest of the body. Their functions are well understood (1). Motor axons conduct action potentials from the brain and spinal cord to skeletal muscles; sensory axons conduct in the opposite direction. All volitional movements and sensations depend on the proper functioning of motor and sensory axons.

Axons are elegantly adapted for their normal functions (Figure 1A and ref. 1). Their cell membrane contains a repertoire of ion channels that generate and propagate action potentials. In addition, axons larger than $1 \mu \mathrm{m}$ in diameter are myelinated by glial cells (Schwann cells and oligodendrocytes in the peripheral and central nervous systems, respectively). Myelin sheaths form segments called internodes around individual axons that are separated by thin gaps (approximately $1 \mu \mathrm{m}$ in length) known as nodes of Ranvier. By both reducing the internodal capacitance and clustering the appropriate ion channels (2), myelination restricts ionic conductance to nodes of Ranvier, enabling action potentials to jump from node to node (saltatory conduction), which is faster and energetically cheaper than continuous conduction.

Their length makes axons vulnerable to disease. Compared with more colossal vertebrates, the human leg is not particularly

Conflict of interest: The author has declared that no conflict of interest exists.

Citation for this article: J Clin Invest. 2011; 121(12):4624-4627. doi:10.1172/JCI60511. long, but a one-meter-long motor axon that innervates the foot presents formidable challenges. Its cell body in the spinal cord not only synthesizes all of the axonal components, it transports them down the axon, a journey that can take up to a year. In addition, the axon requires ATP to maintain its ion gradients and cellular integrity, and this must be locally supplied all along its length.

\section{HSAN1, one of many hereditary peripheral neuropathies}

Damage to peripheral axons that impairs their function results in peripheral neuropathy. Since each peripheral nerve serves a specific part of the body and contains the appropriate axons for that body part, the array of symptoms exhibited by individuals with peripheral neuropathy varies widely. There are also numerous kinds of peripheral neuropathy, and these can be divided into those that are inherited and those that are acquired.

Hereditary peripheral neuropathies are usually called Charcot-Marie-Tooth disease (CMT), in honor of the physicians who first described them. CMT is a common disease, affecting approximately 1 in 2,500 people (3). Patients are classified according to the pattern of inheritance, the degree of clinical severity, the cell type that is primarily affected (myelin sheath or axon/neuron), and - in cases in which neurons are the main affected cell type - the kind of peripheral neuron affected (sensory, motor, and/or autonomic). Mutations in more than 30 genes cause CMT (4), and many more remain to be discovered. The demyelinating forms are the most common, and the genetic causes of most of these conditions have been discovered $(5,6)$. Even though some of the genes associated with demyelinating forms of CMT are expressed in other cell types, the primary abnormality - demyelination - is caused by effects of the mutations in myelinating Schwann cells (7). The axonal forms of CMT are less common, and their genetic causes remain unknown for the majority of affected patients $(5,6)$. In most cases, expression of the mutant gene in the affected neurons likely produces an axonal neuropathy, but this has been experimentally demonstrated for only a few kinds. What both demyelinating and axonal forms of CMT share in common is their tendency to cause a progressive axonal degeneration, beginning with the longest axons (Figure 1, $B$ and $C$ ). The demyelinating forms of CMT typically affect all myelinated sensory and motor axons and spare unmyelinated axons (largely sensory and autonomic axons), whereas the axonal forms of CMT typically affect both myelinated and unmyelinated axons. Some axonal forms, however, chiefly affect either motor axons (called hereditary motor neuropathies) or sensory axons (called hereditary sensory and autonomic neuropathies [HSANs]), including the unmyelinated ones.

Missense mutations in either serine palmitoyltransferase, long chain base subunit 1 $($ SPTLC1 $)(8,9)$ or SPTLC2 $(10)$ - which encode two of the three subunits of the enzyme serine palmitoyltransferase - cause HSAN type 1 (HSAN1), a rare and clinically distinct form of dominantly inherited CMT. HSAN1 is in fact a misnomer because motor axons are typically affected and autonomic defects are sparse (11). Most patients identified to date have a C133W mutation in SPTLC1 and share a common haplotype, and thus a common ancestor (12). These patients most frequently present with decreased sensation in the feet, as well as painless blisters and ulcers. However, positive sensory symptoms, including shooting and burning pain, 
A Healthy peripheral neuron

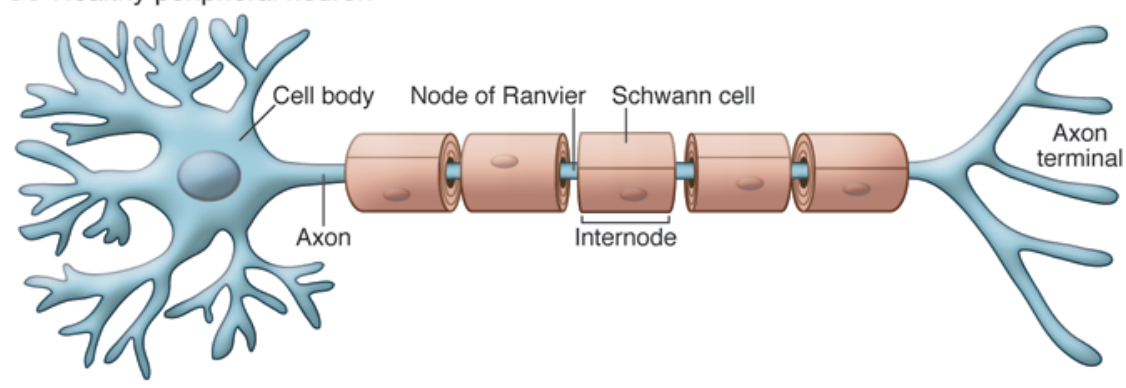

B Inherited demyelinating neuropathy

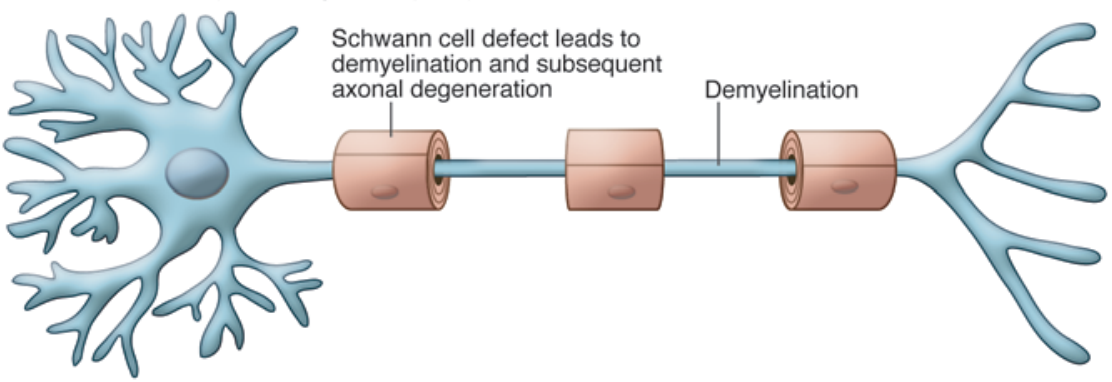

C Inherited axonal neuropathy

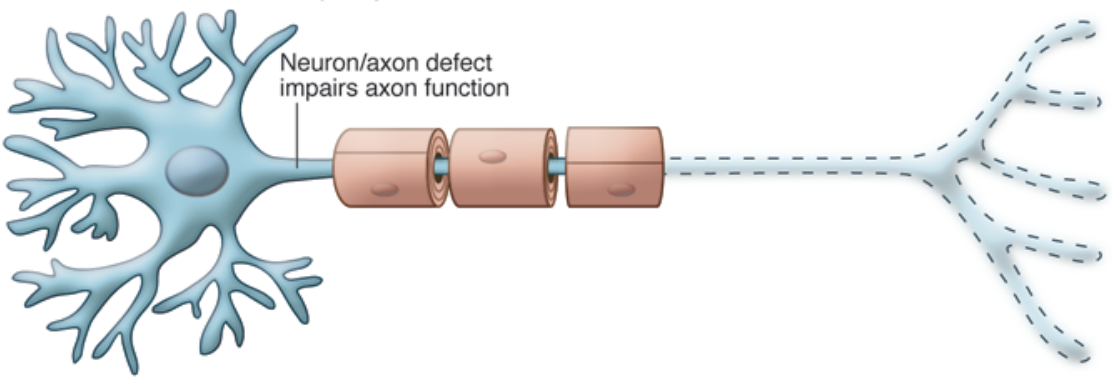

\section{Figure 1}

The effects demyelination and axonal damage on myelinated axons. (A) A single neuron and its axon, which has five myelin internodes that are separated by nodes of Ranvier. (B) In an inherited demyelinating neuropathy, two myelin internodes have been lost, leaving demyelinated segments that will usually be remyelinated. Even though demyelination is the primary pathology, axonal degeneration (see $\mathbf{C}$ ) is a common, long-term consequence. (C) In an inherited axonal neuropathy, the distal part of the axon has degenerated (dashed region), and the myelin sheaths that formerly surrounded the degenerated region have disappeared as a secondary consequence of the axonal degeneration. are also common. Regardless of the initial symptoms, the loss of sensation, especially pain, leads to the horrible complications of unheeded infections and painless ulcers that can result in amputations of the affected extremities (11).

Despite the discrete phenotype arising as a result of these dominant SPTLC1 mutations associated with HSAN1, many cell types, including neurons, express SPTLC1 and SPTLC2 and the enzyme that they form. Serine palmitoyltransferase condenses L-serine with palmitoyl-CoA to form 3-ketosphinganine, which is converted to ceramide, the precursor for complex sphingolipids (Figure 2A). In addition to reducing enzymatic activity, the dominant SPTLC1 and SPTLC2 mutations associated with HSAN1 reduce the substrate specificity of serine palmitoyltransferase, allowing the incorporation of L-alanine and L-glycine and subsequent formation of the atypical deoxysphingolipids 1-deoxysphinganine and 1-deoxymethylsphinganine, respectively (Figure $2 \mathrm{~B}$ and refs. 13, 14). Because they can neither be converted into complex sphingolipids nor degraded by the classical catabolic pathways, deoxysphingolipids accumulate in cells transfected to express the SPTLC1 mutations associated with HSAN1, in transformed lymphoblasts from patients with HSAN1, in tissues (including peripheral nerves) of mice that express the C133W SPTLC1 mutation as a transgene, and in the plasma of patients with HSAN1 (13-15). These data have led to the hypothesis that deoxysphingolipids cause the clinical phenotype of HSAN1.

\section{Toxic lipids cause HSAN1?}

In this issue of the JCI, Garofalo et al. extend their earlier findings indicating that deoxysphingolipids are toxic for cultured sensory neurons (14) by showing that oral supplementation of L-alanine exacerbated the neuropathy of C133W transgenic mice (16). Mice supplemented with L-alanine between 3 and 9 months of age had high- er sensory thresholds than did untreated transgenic mice, and the unmyelinated and myelinated axons in the sciatic nerve tended to have smaller diameters (16). It remains to be rigorously shown, however, that L-alanine exacerbates axonal loss (which should be age and length dependent). Conversely, and even more importantly, Garofalo et al. found that oral supplementation with L-serine improved the neuropathy of C133W transgenic mice (16). Mice treated between 2 and 12 months of age had better rotarod scores than did untreated transgenic mice. In mice treated between 3 and 15 months of age, the diameter of both unmyelinated and myelinated axons increased, the thickness of the myelin sheaths increased, and the number of unmyelinated axons was preserved compared with untreated transgenic mice. As expected, dietary manipulation changed the relative levels of L-alanine and L-serine: C133W transgenic mice fed L-alanine or L-serine had decreased or increased L-serine/ L-alanine ratios, respectively, both in plas- 
A<smiles>CCCCCCCCCCCCCCC(=O)CC(=O)OCC(C)=O</smiles><smiles>CCCCCCCCCCCCCC(O)C(CO)C(N)CO</smiles>

B<smiles>CCCCCCCCCCCCCCC(=O)CC(=O)OCc1ccccc1</smiles><smiles>CCCCCCCCCCCC(O)C(N)CO</smiles>
palmitoyltransferase<smiles>CCCCCCCCCCCCCCC(=O)CC(=O)O</smiles><smiles>Cc1ccccc1</smiles><smiles>CCCCCCCCCCCCCC(O)C(C)N</smiles><smiles>CCCCCCCCCCCCCCCC(=O)CC(=O)O</smiles><smiles>NCC(=O)O</smiles><smiles>CCCCCCCCCCCCCC(O)CN</smiles>

\section{Figure 2}

The effects of SPTLC1 mutations on the synthesis of sphinganine, 1-deoxysphinganine, and 1-deoxymethylsphinganine. (A) SPTLC1, STPLC2, and SPTLC3 form serine palmitoyltransferase, which catalyzes the enzymatic condensation of L-serine and palmitoyl-CoA into sphinganine, a precursor of ceramide. Note that the intermediate, 3-ketosphinganine, is not shown. (B) In the case of SPTLC1 mutants associated with HSAN1, the serine palmitoyltransferase not only catalyzes the enzymatic condensation of L-serine and palmitoyl-CoA into sphinganine, but also catalyzes the enzymatic condensation of palmitoyl-CoA and both L-alanine and L-glycine to generate 1-deoxysphinganine and 1-deoxymethylsphinganine, respectively.

ma and in sciatic nerve. Furthermore, just 3 days of L-serine supplementation decreased the amount of deoxysphingolipids in plasma, to levels comparable to those detected in wild-type mice.

In a pilot study, Garofalo et al. extended these findings to patients with HSAN1 (16). They enrolled 14 patients (aged 24-76 years) who had the C133Y mutation in SPTLC1 and had been symptomatic for 4-52 years. The patients were given 200 or $400 \mathrm{mg} / \mathrm{kg}$ L-serine daily for 10 weeks. No mention is made by the authors of oral L-serine having any side effects, and both doses reduced plasma deoxysphingolipid levels for the duration of the treatment (16). Clinical improvement was not an outcome measure, and would not have been expected owing to the short duration of treatment compared with the long duration of symptoms and the degree of neuropathy.

\section{A clinical trial of L-serine should be done}

The findings of Garofalo et al. (16) are an important advance for individuals who have HSAN1, and the news of a potential treatment for CMT will reverberate around the CMT community. There will be many phone calls and e-mails to answer from patients who have other forms of CMT, and even other kinds of neuropathy, so let me anticipate the answers to some of their questions here: L-serine is unlikely to help patients who have other forms of neuropathy, including other forms of CMT; L-serine has not yet been proven to help patients who have HSAN1. It will be difficult to perform such a clinical trial - the number of HSAN1 patients is small, the natural history of HSAN1 is incompletely known, there are no validated outcome measures for HSAN1, and it is unlikely that any therapy can reverse the ravages of a chronic neuropathy. L-serine may be a rational therapy for HSAN1, but that does not guarantee that it will prevent its progression. If L-serine proves to be an effective treatment for HSAN1, then it will be appropriate to screen more patients who have the HSAN1 phenotype for SPTLC1 and SPTLC2 mutations, in order to help as many patients as possible.

\section{Some pieces of the puzzle are still missing}

Despite the advances toward a potential therapy for HSAN1 made by Garofalo et al. (16), unraveling the cause of neuropathy in individuals with HSAN1 will require more study. As the authors discuss, it is not clear which compounds produce neurotoxicity or why neurons and their axons are selectively affected when SPTLC1 is expressed 
in many cell types. Nor is the issue of cell autonomy settled: does the expression of SPTLC1 mutations by neurons alone lead to neuropathy? The $\mathrm{C} 133 \mathrm{~W}$ transgenic mice used by Garofalo et al. (16) are not helpful in this regard, because expression of the C133W mutation is controlled by the $\beta$-actin promoter, which is ubiquitously expressed. Furthermore, even with this transgene, the amount of measured axonal loss is slight compared with the devastating loss that has been documented in affected patients (11). Let's get back to the lab.

\section{Acknowledgments}

The author's work is supported by the NIH, the National Multiple Sclerosis Society, the Muscular Dystrophy Association, and the Charcot-Marie-Tooth Association.

Address correspondence to: Steven S. Scherer, Department of Neurology, The Perelman School of Medicine at the University of Pennsylvania, Room 450 Stemmler Hall, 36th Street and Hamilton Walk, Philadelphia, Pennsylvania 19104-6077, USA.
Phone: 215.573.3198; Fax: 215.573.4454;

E-mail: sscherer@mail.med.upenn.edu.

1. Kandel ER, Schwartz JP, Jessell TM. Principles of Neural Science. New York, New York, USA: Elsevier; 2000

2. Salzer JL. Polarized domains of myelinated axons. Neuron. 2003;40(2):297-318

3. Shy ME, Lupski JR, Chance PF, Klein CJ, Dyck PJ. Hereditary motor and sensory neuropathies: an overview of clinical, genetic, electrophysiologic, and pathologic features. In: Dyck PJ, Thomas PK, eds. Peripheral Neuropathy. Philadelphia, Pennsylvania, USA: Saunders; 2005:1623-1658.

4. Timmerman V. Inherited Peripheral Neuropathies Mutation Database. Human Genome Variation Society web site. http://www.molgen.ua.ac. be/CMTMutations/. Updated February 17, 2011. Accessed September 23, 2011.

5. Latour P, et al. SIMPLE mutation analysis in dominant demyelinating Charcot-Marie-Tooth disease: three novel mutations. J Peripher Nerv Syst. 2006:11(2):148-155.

6. Saporta ASD, Sottile SL, Miller LJ, Feely SME, Siskind CE, Shy ME. Charcot-Marie-Tooth disease subtypes and genetic testing strategies. Ann Neurol. 2011;69(1):22-33

7. Scherer SS, Wrabetz L. Molecular mechanisms of inherited demyelinating neuropathies. Glia. 2008;56(14):1578-1589

8. Bejaoui K, et al. SPTLC1 is mutated in hereditary sensory neuropathy, type 1. Nat Genet. 2001; 27(3):261-262.

9. Dawkins JL, Hulme DJ, Brahmbhatt SB, Auer-
Grumbach M, Nicholson GA. Mutations in SPTLC1, encoding serine palmitoyltransferase, long chain base subunit-1, cause hereditary sensory neuropathy type I. Nat Genet. 2001;27(3):309-312.

10. Rotthier A, et al. Mutations in the SPTLC2 subunit of serine palmitoyltransferase cause hereditary sensory and autonomic neuropathy type I. Amer J Hum Genet. 2010;87(4):513-522.

11. Houlden $\mathrm{H}$, et al. Clinical, pathological and genetic characterization of hereditary sensory and autonomic neuropathy type 1 (HSAN I). Brain. 2006; 129(pt 2):411-425.

12. Nicholson GA, Dawkins JL, Blair IP, AuerGrumbach M, Brahmbhatt SB, Hulme DJ. Hereditary sensory neuropathy type I: Haplotype analysis shows founders in southern England and Europe. Amer J Hum Genet. 2001;69(3):655-659.

13. Gable K, Gupta SD, Han G, Niranjanakumari S, Harmon JM, Dunn TM. A disease-causing mutation in the active site of serine palmitoyltransferase causes catalytic promiscuity. J Biol Chem. 2010;285(30):22846-22852.

14. Penno A, et al. Hereditary sensory neuropathy type 1 is caused by the accumulation of two neurotoxic sphingolipids. J Biol Chem. 2010;285(15):11178-11187.

15. Eichler FS, et al. Overexpression of the wild-type SPT1 subunit lowers desoxysphingolipid levels and rescues the phenotype of HSAN1. J Neurosci. 2009;29(46):14646-14651

16. Garofalo K, et al. Oral L-serine supplementation reduces production of neurotoxic deoxysphingolipids in mice and humans with hereditary sensory autonomic neuropathy type 1 . J Clin Invest. 2011;121(12):4735-4745

\title{
Another VCP interactor: NF is enough
}

\author{
Conrad C. Weihl
}

Department of Neurology and Hope Center on Neurological Disorder, Washington University School of Medicine, Saint Louis, Missouri, USA.

\begin{abstract}
Inclusion body myopathy with Paget disease of the bone and frontotemporal dementia (IBMPFD) is a multisystem degenerative disorder caused by mutations in the valosin-containing protein $(V C P)$ gene. How missense mutations in this abundant, ubiquitously expressed, multifunctional protein lead to the degeneration of disparate tissues is unclear. VCP participates in diverse cellular functions by associating with an expanding collection of substrates and cofactors that dictate its functionality. In this issue of the JCI, Wang and colleagues have further expanded the VCP interactome by identifying neurofibromin-1 (NF1) as a novel VCP interactor in the CNS. IBMPFD-associated mutations disrupt binding of VCP to NF1, resulting in reduced synaptogenesis. Thus, aberrant interactions between VCP and NF1 may explain the dementia phenotype and cognitive delay observed in patients with IBMPFD and neurofibromatosis type 1.
\end{abstract}

Inclusion body myopathy with Paget disease of the bone and frontotemporal dementia (IBMPFD) is a rare, late ageonset inherited degenerative disorder that can affect the muscles, bones, and brain. It is caused by autosomal dominant muta-

Conflict of interest: The author has declared that no conflict of interest exists.

Citation for this article: J Clin Invest. 2011; 121(12):4627-4630. doi:10.1172/JCI61126. tions in the valosin-containing protein (VCP; also known as p97) gene. Although IBMPFD is a fully penetrant disorder, it has variably penetrant phenotypes (1). Approximately $50 \%$ of IBMPFD patients initially present with muscle weakness in mid-life, and more than $90 \%$ will eventually develop disabling weakness. Characteristic of the heterogeneity of this disorder, patients can initially present with a proximal or distal pattern of weakness that is often asymmet- ric. As the disease progresses, patients lose their ability to ambulate and develop respiratory failure that ultimately leads to death within 10-15 years of initial presentation. Among IBMPFD patients, 50\% develop Paget disease of the bone (PDB), manifesting as painful osteolytic lesions, and 35\% develop frontotemporal dementia (FTD), with mean onset $7-10$ years later than muscle weakness or PDB. The potential for progressing to FTD is particularly troubling to patients, who may experience changes in personality, language abilities, as well as cognitive function. The phenotypic diversity of IBMPFD is not attributable to different mutations, since two patients with the same VCP point mutation, even within the same family, can present with drastically different clinical features (e.g., dementia in one and isolated muscle weakness in another) (2). This intrafamilial variability makes the diagnosis of IBMPFD difficult. Whether there are environmental or genetic modifiers that explain this phenotypic variability is not known. 